\title{
Anel corneano intra-estromal para baixa miopia - Estudo comparativo com a técnica de LASIK
}

\author{
Intrastromal corneal ring for low myopia - A comparative study with LASIK
}

\author{
Adriana dos Santos Forseto ${ }^{1}$ \\ Paulo Schor ${ }^{2}$ \\ Rubens Belfort $\mathbf{J r}^{3}$ \\ Walton Nosé ${ }^{4}$
}

Trabalho realizado na Universidade Federal de São Paulo - UNIFESP.

Doutora em Medicina pela Universidade Federal de São Paulo - UNIFESP e médica do Eye Clinic Day Hospital. ${ }^{2}$ Doutor em Medicina pela Universidade Federal de São Paulo - UNIFESP.

${ }^{3}$ Professor Livre Docente e Titular de Oftalmologia da Universidade Federal de São Paulo - UNIFESP.

${ }^{4}$ Professor Livre Docente pela Universidade Federal de São Paulo - UNIFESP, Professor Titular de Oftalmologia da Universidade Metropolitana de Santos - UNIMES, Médico do Eye Clinic Day Hospital.

Endereço para correspondência: Adriana dos Santos Forseto, Av. República do Líbano 1034 - São Paulo (SP) CEP 04502-001

E-mail: forseto@uol.com.br

Recebido para publicação em 11.04.2003

Versão revisada recebida em 28.08.2003

Aprovação em 19.09.2003

Nota Editorial: Pela análise deste trabalho e por sua anuência na divulgação desta nota, agradecemos aos Drs. Hamilton Moreira, Paulo Ricardo de Oliveira e Hélia Soares Angotti.

\begin{tabular}{|l|}
\hline RESUMO \\
\hline Objetivo: Avaliar prospectivamente a eficácia e segurança dos segmentos \\
de anéis corneanos intra-estromais (ICRS) para a correção de baixa miopia \\
e comparar os resultados obtidos com a técnica de laser in situ keratomi- \\
leusis (LASIK). Métodos: Estudo prospectivo, bilateral e não randomizado. \\
Quatorze pacientes com equivalente esférico entre -1,00 e -4,50 D foram \\
submetidos ao implante de ICRS em um olho è técnica de LASIK no olho \\
contralateral. As avaliações pós-operatórias foram realizadas com um e \\
sete dias, um, três, seis, 12e24meses. Resultados: Aoúltimo exame, 64,29\% \\
dos olhos (9/14) do grupo ICRS e 85,71\% (12/14) após o LASIK estavam \\
entre $\pm 0,50$ D da correção desejada (p=0,385). Acuidade visual sem \\
correção $\geq 20 / 20$ foi obtida em quatro dos 14 olhos (28,57\%) do grupo ICRS, \\
e em 12 dos 14 olhos (85,71\%) do grupo LASIK (p=0,002*). Durante o \\
seguimento, não foram observadas variações refracionais superiores a \\
1,00 D, entre dois exames consecutivos, em ambos os grupos, ou perda \\
significante da melhor acuidade visual corrigida. Maior indução de astig- \\
matismo foi encontrada nos olhos implantados com ICRS. Ao exame de 24 \\
meses, o grupo ICRS apresentou valores inferiores no exame de sensibi- \\
lidade ao contraste, especialmente nas frequiências espaciais mais altas \\
(p=0,032* em 18 cpd). Foram relatadas mais queixas subjetivas no período \\
pós-operatório precoce de ICRS em relação ao LASIK. Conclusões: Os \\
ICRS foram considerados eficazes na correção de baixa miopia, porém sua \\
segurança foi limitada pela indução de astigmatismo no pós-operatório, e \\
seus resultados foram inferiores aos do grupo controle.
\end{tabular}

Descritores: Miopia/cirurgia; Córnea/cirurgia; Implante de prótese; Ceratomileuse assistida por excimer laser in situ; Miopia/classificação

\section{INTRODUÇ̃̃̃O}

A utilização de implantes sintéticos intracorneanos para a correção de erros refrativos foi inicialmente idealizada por Barraquer em 1949, que introduziu o termo Ceratoplastia Refrativa, ou seja, uma cirurgia plástica na córnea com finalidade refrativa ${ }^{(1)}$.

Nos anos subseqüentes, várias pesquisas foram feitas na tentativa de se encontrar algum material que fosse biocompatível, permeável e apresentasse boa qualidade óptica e alto índice de refração ${ }^{(2-4)}$.

Todos estes métodos envolviam a disrupção do estroma corneano centralmente no eixo visual. Posteriormente foi sugerida a possibilidade de

\footnotetext{
* Significante
} 
correção da miopia por meio do aumento da espessura da periferia da córnea ${ }^{(5)}$. Em 1987 foi descrito um implante intraestromal de polimetilmetacrilato (PMMA), o anel corneano intra-estromal ("Intrastromal corneal ring" ou ICR), cuja localização periférica permitia a manutenção de uma região central da córnea fisiologicamente intacta ${ }^{(6-7)}$.

Os primeiros implantes de ICR em olhos não funcionais e funcionais de seres humanos foram iniciados em 1991 por Nosé no Brasil ${ }^{(8-9)}$, seguidos por Assil nos Estados Unidos ${ }^{(10)}$. Em 1995, durante a fase II de estudos clínicos do FDA (Food and Drug Admnistration) norte-americano, foi introduzido um anel de desenho diferente, os "Intrastromal corneal ring segments" (ICRS) ${ }^{(11)}$. Em abril de 1999, estes segmentos, agora conhecidos como "Intacs", foram aprovados pelo FDA para correção de miopia entre -1,00 e -3,00 dioptrias (D).

Os anéis corneanos intra-estromais foram desenhados na tentativa de melhorar os resultados obtidos com a cirurgia refrativa corneana por meio da redução dos efeitos da cicatrização da córnea e manutenção de sua asfericidade. Além disso, a técnica é considerada reversível e ajustável, melhorando desta forma o resultado refracional ${ }^{(8-9,12-14)}$.

Até o momento, várias publicações reportaram os resultados da técnica de implante dos anéis ou segmentos de anéis corneanos intra-estromais para a correção da baixa miopia ${ }^{(8-11,15-19)}$, mas não de forma controlada. Outros estudos realizaram comparações com as técnicas de excimer laser, como a PRK (ceratectomia fotorrefrativa) ${ }^{(20)}$ ou o LASIK (excimer laser associado à técnica de ceratomileuse) $)^{(21)}$, mas não prospectivamente.

Este estudo tem como objetivo avaliar prospectivamente a eficácia e segurança dos ICRS para a correção de baixa miopia e comparar os resultados obtidos com um grupo controle tratado pela técnica de laser in situ keratomileusis (LASIK).

\section{MÉTODOS}

Este estudo clínico prospectivo, controlado, realizado sob coordenação do Departamento de Oftalmologia da Universidade Federal de São Paulo, foi analisado e aprovado pelo Comitê de Ética Médica do Hospital São Paulo, tendo sido obtido termo de consentimento assinado de todos os participantes.

Os implantes intracorneanos utilizados neste estudo (ICRS, KeraVision, Inc., Califórnia, EUA) são compostos por dois segmentos de anéis de PMMA, cada qual com $150^{\circ}$ de comprimento de arco. Apresentam secção hexagonal sobre uma plataforma cônica e espessura variável de 0,25 a $0,45 \mathrm{~mm}$, em incrementos de $0,05 \mathrm{~mm}$.

Quatorze pacientes, com idade entre 21 e 50 anos (média 33,25 $\pm 9,23$ anos) foram selecionados para serem submetidos à cirurgia de implante de segmentos de anéis corneanos intra-estromais em um de seus olhos (grupo ICRS, $\mathrm{n}=14$ ). O grupo controle foi constituído pelos olhos contralaterais destes pacientes operados pela técnica de LASIK ( $\mathrm{n}=14$ ), em média 6,27 $\pm 1,94$ meses (variação: 3 a 8 meses) após o procedimento inicial.

Todos tinham acuidade visual corrigida de $20 / 20$, ou melhor, refração estável com equivalente esférico entre -1,00 e -4,50 D, e astigmatismo menor ou igual a 1,00 D no olho a ser submetido ao implante de ICRS. Usuários de lentes de contato foram orientados a suspender o seu uso pelo período mínimo de 2 a 3 semanas.

Foram excluídos pacientes gestantes ou com sinais de doenças sistêmicas que pudessem aumentar seu risco cirúrgico ou alterar a cicatrização da córnea, além de condição ocular prévia que pudesse predispor a futuras complicações como ceratocone, distrofias corneanas, glaucoma, alteração do filme lacrimal ou história de herpes ocular. Outros critérios de exclusão foram: diferenças de equivalente esférico entre os dois olhos de 1,00 D ou mais, curvatura corneana central superior a $46,00 \mathrm{D}$ ou inferior a $40,00 \mathrm{D}$, paquimetria menor que $0,48 \mathrm{~mm}$ na região central ou $0,57 \mathrm{~mm}$ na periférica.

A avaliação oftalmológica foi realizada no período pré e pós-operatório de um e sete dias, um, três, seis, 12 e 24 meses. Esta incluiu medidas de acuidade visual para longe com e sem a melhor correção, utilizando-se as tabelas de acuidade visual do ETDRS (Early Treatment Diabetic Retinopathy Study), refração dinâmica e estática, biomicroscopia em lâmpada de fenda, sensibilidade ao contraste com correção (aparelho CSV-1000, VectorVision, Ohio, EUA), videoceratografia computadorizada (EyeSys Corneal Analysis System - Eye Sys Technologies, Dallas, EUA) e paquimetria ultra-sônica (Ocuscan, Alcon, Texas, EUA). A pesquisa de queixas subjetivas, como ofuscamento e visão de halos, e da satisfação e preferência dos pacientes foi realizada por meio de um questionário elaborado para o estudo.

Os critérios de eficácia e segurança utilizados foram semelhantes aos definidos durante as fases II e III de estudos clínicos dos ICRS pelo FDA ${ }^{(16)}$. A eficácia em relação à previsibilidade foi demonstrada pela obtenção do efeito refrativo dentro da faixa de $\pm 1,00 \mathrm{D}$ da correção desejada em $75 \%$ dos olhos, ou entre $\pm 0,50 \mathrm{D}$ em $50 \%$, por meio da análise do equivalente esférico da refração cicloplegiada. A eficácia em relação à estabilidade foi considerada frente à variação $\leq 1,00 \mathrm{D}$ entre dois exames consecutivos com intervalo mínimo de três meses, a partir do terceiro mês pós-operatório, em $95 \%$ dos olhos tratados; ou $\leq 0,50 \mathrm{D}$ em $75 \%$ dos casos. A observação de acuidade visual não corrigida (AV s/c) pós-operatória melhor ou igual a 20/40 em pelo menos $85 \%$ dos olhos foi definida como critério de eficácia na acuidade visual. A presença de efeitos adversos e/ou complicações oculares, como perda de duas ou mais linhas da melhor acuidade visual corrigida $(\mathrm{AV} \mathrm{c} / \mathrm{c})$ e indução de astigmatismo $\geq 1,00 \mathrm{D}$, foram avaliadas para análise da segurança.

Todas as cirurgias foram realizadas pelo mesmo cirurgião (WN), sob anestesia tópica, após instilação de colírio de cloridrato de proximetacaína a $0,5 \%$ (Anestalcon ${ }^{\circledR} 0,5 \%$, Alcon Laboratórios do Brasil Ltda., São Paulo, Brasil), no período compreendido entre outubro de 1998 e agosto de 1999.

A escolha do ICRS a ser implantado foi baseada no equivalente esférico da refração sob cicloplegia, conforme nomograma clínico fornecido pelo fabricante (Tabela 1). Centralizou-se um instrumento especial de marcação (KeraVision, Inc., Cali- 


\begin{tabular}{|c|c|c|c|c|}
\hline $\begin{array}{l}\text { ICRSKeraVision }^{\circledR} \\
\text { Espessura } \\
(\mathrm{mm})\end{array}$ & $\mathbf{n}$ & $\begin{array}{l}\text { Correção } \\
\text { prevista } \\
\text { (D) }\end{array}$ & $\begin{array}{l}\text { Correção } \\
\text { média obtida } \\
\text { (D) }\end{array}$ & $\begin{array}{l}\text { Desvio- } \\
\text { padrão }\end{array}$ \\
\hline 0,25 & 1 & $-1,3$ & $-1,37$ & 0,00 \\
\hline 0,30 & 5 & $-2,0$ & $-1,72$ & 0,18 \\
\hline 0,35 & 2 & $-2,7$ & $-1,43$ & 0,61 \\
\hline 0,40 & 4 & $-3,4$ & $-2,71$ & 0,50 \\
\hline 0,45 & 2 & $-4,1$ & $-3,31$ & 0,44 \\
\hline
\end{tabular}

fórnia, EUA) sobre o centro geométrico da córnea, sendo este responsável pelo correto posicionamento da incisão radial periférica, aproximadamente a $4 \mathrm{~mm}$ da região central. Uma incisão radial de cerca de 1,8 mm de extensão foi realizada, na posição das 12 horas, com um bisturi de diamante calibrado para um corte de $68 \%$ do valor da paquimetria intra-operatória daquele local. A seguir, um separador estromal de Suarez (Storz, Missouri, EUA) foi inserido na base da incisão e o tecido corneano foi separado lateralmente, a fim de preparar a entrada para o divulsionador estromal. Na seqüência, um dispositivo de fixação do bulbo ocular a vácuo foi aplicado à conjuntiva perilimbar, para servir de guia para os instrumentos de dissecção estromal (KeraVision, Inc., Califórnia, EUA), que foram inseridos na base da incisão e rodados nos sentidos horário e anti-horário a fim de separar as lamelas corneanas em camadas e criar dois semicanais. Após a criação dos canais estromais, o dispositivo de fixação a vácuo foi removido, procedendo-se com o implante dos segmentos, sendo a incisão suturada com um ponto de mononylon 10-0 (Alcon Laboratórios do Brasil, São Paulo, Brasil). Das 14 cirurgias de ICRS realizadas, as seis primeiras não foram suturadas.

A preparação do disco corneano e leito estromal da cirurgia de LASIK do olho contralateral foi realizada com o microcerátomo Automated Corneal Shaper (Chiron Ophthalmics ${ }^{\circledR}$ Inc., Califórnia, EUA), com plataforma de $160 \mu$ m e diâmetro de $8,5 \mathrm{~mm}$. A fotoablação corneana foi centralizada na pupila de entrada do paciente e feita com o aparelho de "excimer laser" ChironTechnolas 217C - versão 2.9992 (Bausch \& Lomb Surgical Inc., New York, EUA). Programou-se a correção total do erro refracional (componente esférico e cilíndrico) baseando-se na refração cicloplegiada pré-operatória, utilizando-se uma zona de tratamento de 5,0 mm em todos os casos, e zona de transição que variou entre 7,9 e 11,2 $\mathrm{mm}$. A profundidade de ablação variou de 20 a $75 \mu \mathrm{m}$ (média: 45,28 $\pm 15,22 \mu \mathrm{m}$ ), conforme a correção programada.

A medicação pós-operatória consistiu de colírio de tobramicina a $0,3 \%$ em associação com dexametasona $0,1 \%$ (Tobradex ${ }^{\circledast}$, Alcon Laboratórios do Brasil, São Paulo, Brasil), por 4 vezes ao dia, com regressão até completa suspensão ao término da segunda semana.

As variáveis quantitativas foram representadas por média, desvio padrão (D.P.), mediana, valores mínimo e máximo. Para fins de análise estatística, as medidas de acuidade visual foram calculadas em LogMAR. Os valores de sensibilidade ao contraste foram convertidos em unidades de log, conforme tabela fornecida pelo fabricante. As variáveis qualitativas foram representadas por freqüência absoluta (n) e relativa (\%). Foram aplicados testes paramétricos e não paramétricos. Adotou-se o nível de significância de $0,05(\alpha=5 \%)$. Níveis descritivos (p) inferiores a esse valor foram considerados significantes e representados por*. Utilizou-se o programa de computador SPSS 8.0 for Windows. A análise dos dados de astigmatismo foi feita de acordo com o método vetorial de Alpins ${ }^{(22)}$, tendo-se calculado os vetores da correção desejada de astigmatismo ( "target induced astigmatism "ou TIA) e do astigmatismo induzido cirurgicamente ("surgically induced astigmatism" ou SIA). A partir do diferencial destes vetores foi possível a obtenção da média e desvio-padrão da magnitude de erro, cujo valor próximo de zero indica o sucesso do procedimento cirúrgico.

\section{RESULTADOS}

Todos os olhos $(\mathrm{n}=28)$ foram acompanhados em todas visitas pós-operatórias propostas, com seguimento completo de 24 meses. Não foram observadas diferenças estatisticamente significantes entre os dois grupos em relação às características oftalmológicas avaliadas no pré-operatório como, por exemplo, equivalente esférico da refração dinâmica $(\mathrm{p}=0,571)$ e estática $(\mathrm{p}=0,701)$, astigmatismo $(\mathrm{p}=0,946)$, acuidade visual sem correção média $(\mathrm{p}=0,541)$, ceratometria média $(\mathrm{p}=0,874)$ e sensibilidade ao contraste nas diferentes freqüências espaciais ( 3 cpd: $\mathrm{p}=0,165 ; 6 \mathrm{cpd}$ : $\mathrm{p}=0,487 ; 12 \mathrm{cpd}$ : $\mathrm{p}=0,139 ; 18 \mathrm{cpd}$ : $\mathrm{p}=0,096$ ).

Todos os procedimentos cirúrgicos foram realizados sem intercorrências, não tendo sido observadas complicações peroperatórias em ambos os grupos. As cirurgias de implante de ICRS duraram em média 10,15 $\pm 2,19$ minutos (variação de sete a 14 minutos), e as de LASIK, 5,64 $\pm 0,84$ minutos (variação de cinco a sete minutos).

Os implantes utilizados no grupo ICRS, assim como as correções previstas (conforme nomograma) e médias obtidas ao exame de 24 meses, baseadas no equivalente esférico da refração estática, estão discriminados na tabela 1 .

\section{Refração}

No grupo ICRS o equivalente esférico médio da refração dinâmica foi reduzido de $-2,82 \pm 0,84 \mathrm{D}$ (variação de $-4,38 \mathrm{D}$ a $-1,25 \mathrm{D}$ ) para $-0,61 \pm 0,46 \mathrm{D}$ (variação de $-1,50 \mathrm{D}$ a plano) ao exame de 24 meses $(\mathrm{p}<0,001 *)$, não tendo sido observadas diferenças estatisticamente significantes entre os diversos momentos pós-operatórios ( $\mathrm{p}>0,05)$.

O grupo controle apresentou uma redução do equivalente esférico médio da refração dinâmica de $-2,66 \pm 1,00$ D no préoperatório (variando de $-4,25$ a $-1,25 \mathrm{D}$ ) para $-0,29 \pm 0,26 \mathrm{D}$ no pós-operatório de 24 meses, com variação de $-0,75 \mathrm{D}$ a plano $(\mathrm{p}<0,001 *)$. Discreta tendência à regressão do efeito refrativo, sem significância estatística, foi observada a partir da primeira semana de pós-operatório (Figura 1). 
A figura 1 mostra a evolução da média do equivalente esférico da refração dinâmica durante todo o seguimento de 24 meses em ambos os grupos. Diferenças entre eles foram observadas nos momentos de um dia $\left(\mathrm{p}<0,001^{*}\right)$, uma semana $(\mathrm{p}<0,001 *)$, um mês $(\mathrm{p}=0,004 *)$, três meses $\left(\mathrm{p}=0,031^{*}\right)$, e seis meses $\left(\mathrm{p}=0,027^{*}\right)$.

A partir da primeira semana de pós-operatório não foram observadas variações no equivalente esférico da refração dinâmica superiores a 1,00 D, em dois exames consecutivos, em ambos os grupos.

Aos 24 meses de pós-operatório, 64,29\% dos olhos (9/14) do grupo ICRS e $85,71 \%$ dos olhos (12/14) do grupo controle apresentavam equivalente esférico da refração estática dentro da faixa de $\pm 0,50 \mathrm{D}$ da correção desejada $(\mathrm{p}=0,385)$. Valores entre $\pm 1,00 \mathrm{D}$ foram obtidos por $78,57 \%$ (11/14) e $100 \%$ dos casos (14/14) dos grupos ICRS e controle respectivamente $(\mathrm{p}=0,222)$.

Foi encontrada correlação negativa estatisticamente significante entre a espessura dos segmentos de anéis implantados e o equivalente esférico da refração estática ao exame de 24 meses $\left(\mathrm{r}=-0,61384 ; \mathrm{p}=0,0195^{*}\right)$.

Os dois grupos não apresentaram diferenças em relação ao astigmatismo nos períodos pré $(\mathrm{p}=0,946)$ e pós-operatório de 24 meses $(p=0,100)$. Diferenças foram observadas durante os exames de um dia $\left(\mathrm{p}<0,001^{*}\right)$, uma semana $\left(\mathrm{p}<0,001^{*}\right)$, um mês $(\mathrm{p}<0,001 *)$, três meses $(\mathrm{p}=0,002 *)$, seis meses $(\mathrm{p}=0,003 *)$, e 12 meses $\left(\mathrm{p}=0,007^{*}\right)$. A figura 2 mostra a evolução da média do astigmatismo da refração dinâmica durante todo o seguimento de 24 meses.

Pelo método de análise vetorial, os vetores da correção desejada de astigmatismo ("target induced astigmatism" ou TIA) para todo o grupo ICRS foram considerados iguais a zero, visto que o objetivo do procedimento era manter o astigmatismo inalterado nestes olhos. Ao exame de 24 meses, oito casos $(57,14 \%)$ do grupo ICRS apresentaram vetor do astigmatismo induzido

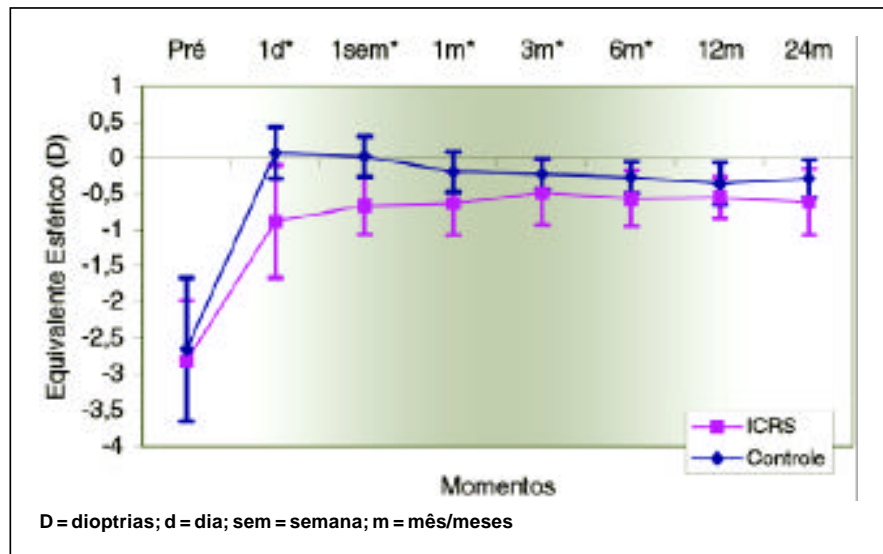

Figura 1 - Evolução da média do equivalente esférico da refração dinâmica, com desvio-padrão, ao longo de todo o estudo: comparação entre os olhos submetidos ao implante de ICRS $(n=14)$ e os controles $(n=14)$, operados pela técnica de LASIK. Diferenças estatisticamente significantes foram representadas por $\left({ }^{*}\right)$ cirurgicamente ("surgically induced astigmatism" ou SIA) menor ou igual a 0,50 ; três casos $(21,42 \%)$ entre 0,51 e 1,00 ; e o restante ( $\mathrm{n}=3$ ou $21,42 \%$ ) entre 1,01 e 1,50 (Figura 3 ). Os valores da magnitude de erro, calculados pela diferença vetorial entre o SIA e a TIA, foram equivalentes ao SIA, com média de $1,61 \pm 0,78 \mathrm{D}$ (variação de 0,36 a 2,89 D) no período pós-operatório de 1 semana e de $0,67 \pm 0,34 \mathrm{D}$ (variação de 0,26 a 1,36 D) ao último exame ( $\left.\mathrm{p}<0,05^{*}\right)$.

Foi observada correlação positiva entre a espessura dos segmentos de anéis corneanos intra-estromais implantados e os vetores dos astigmatismos induzidos cirurgicamente em todos os momentos pós-operatórios avaliados, com significância estatística aos exames de uma semana $\left(\mathrm{p}<0,001^{*}\right)$, um mês $(\mathrm{p}=0,01 *)$ e 12 meses $(\mathrm{p}=0,01 *)$.

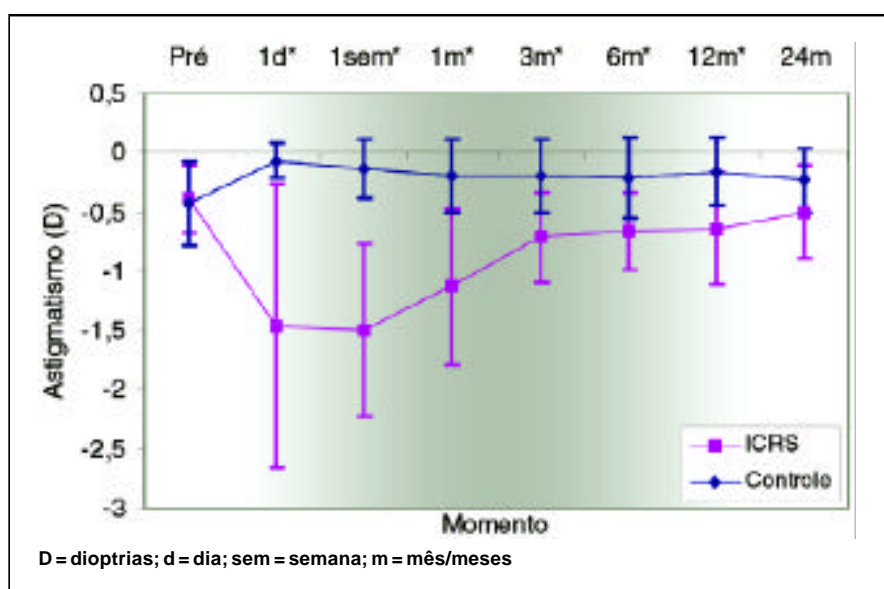

Figura 2 - Evolução da média do astigmatismo da refração dinâmica, com desvio-padrão: comparação entre os olhos submetidos ao implante de ICRS $(n=14)$ e os controles, operados pela técnica de LASIK $(n=14)$. Diferenças estatisticamente significantes entre os dois grupos foram representadas por $\left(^{*}\right)$

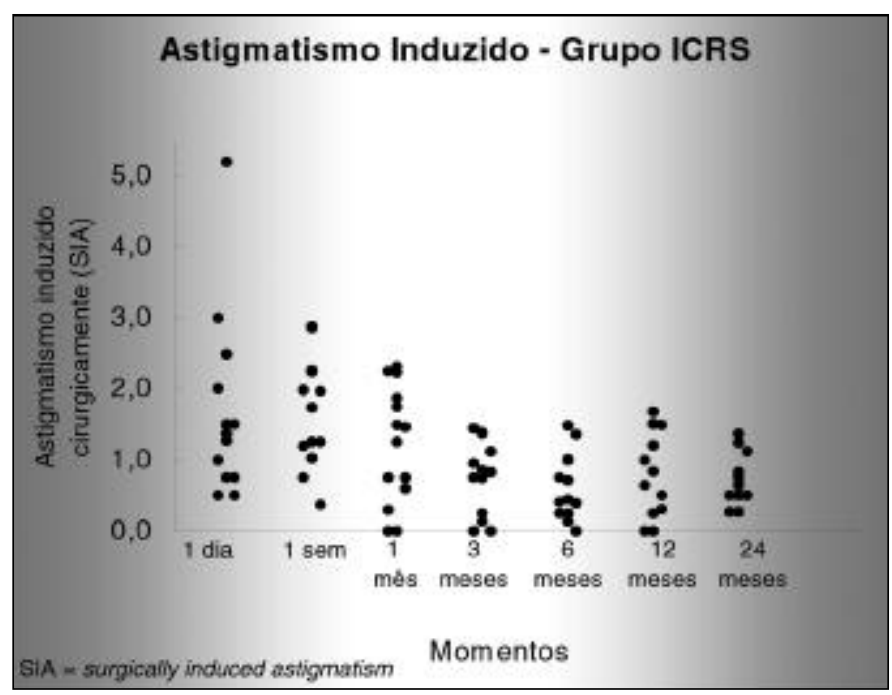

Figura 3 - Diagrama de dispersão do astigmatismo induzido cirurgicamente nos olhos do grupo ICRS ao longo de todo o seguimento pósoperatório $(n=14)$, de acordo com o método de análise vetorial 
No grupo controle, os vetores da correção desejada de astigmatismo variaram entre 0,00 e 1,25 , com média de $0,39 \pm 0,37$, valor este estatisticamente diferente do grupo ICRS $\left(\mathrm{p}=0,0030^{*}\right)$. A média do astigmatismo induzido cirurgicamente manteve-se estável durante todo o seguimento pós-operatório $(\mathrm{p}=0,1450)$. Ao último exame, oito casos $(57,14 \%)$ apresentaram vetor do astigmatismo induzido cirurgicamente menor ou igual a 0,50 ; quatro casos $(28,57 \%)$ entre 0,51 e 1,00 ; e o restante $(n=2$ ou $14,28 \%$ ) entre 1,01 e 1,50 .

Os valores da magnitude de erro do grupo controle mantiveram-se estáveis durante todo o seguimento pós-operatório $(\mathrm{p}=0,1450$ ), com média de $0,18 \pm 0,22 \mathrm{D}$ (variação de $0,00 \mathrm{a} 0,59 \mathrm{D}$ ) ao exame de 24 meses. Seus valores máximos sempre foram inferiores a $1,00 \mathrm{D}$.

Diferenças entre os dois grupos foram observadas em relação à magnitude de erro em todos os momentos avaliados (um dia: $\mathrm{p}<0,001^{*}$; uma semana: $\mathrm{p}<0,001^{*}$; um mês: $\mathrm{p}=0,0002^{*}$; três meses: $\mathrm{p}=0,0042 *$; seis meses: $\mathrm{p}=0,0049^{*} ; 12$ meses: $\mathrm{p}=0,0014^{*}$; e 24 meses: $\mathrm{p}=0,0001 *$ ).

\section{Acuidade visual sem correção}

No grupo ICRS, a média da AV s/c (em LogMAR) era de $0,96 \pm 0,14$ (com variação de 0,70 a 1,20 ) no pré-operatório e de $0,16 \pm 0,19$ (variando entre $-0,09$ e 0,60 ) ao exame de 24 meses $\left(\mathrm{p}<0,001^{*}\right)$, não tendo sido observadas diferenças estatisticamente significantes entre os diversos momentos pós-operató$\operatorname{rios}(\mathrm{p}>0,05)$.

No grupo controle, as médias de AV s/c (LogMAR) encontradas nos exames pré e pós-operatório de 24 meses foram de $0,94 \pm 0,12$ (variação: 0,70 a 1,20), e 0,00 $\pm 0,07$ (variação: $-0,09$ a 0,20$)$, respectivamente $(p<0,001 *)$. Os momentos pós-operatórios não diferiram entre si $(\mathrm{p}>0,05)$.

Diferenças entre os dois grupos foram observadas nos exames de um dia $(\mathrm{p}<0,001 *)$; uma semana $(\mathrm{p}<0,001 *)$; um mês $(\mathrm{p}=0,002 *), 12$ meses $(\mathrm{p}=0,012 *)$ e 24 meses $\left(\mathrm{p}=0,021^{*}\right)$.

No primeiro dia pós-operatório, um maior número de olhos do grupo controle apresentava níveis de AV s/c $\geq 20 / 20 \mathrm{em}$ comparação ao grupo ICRS (11 ou 78,57\% contra um ou 7,14\%, respectivamente) $(\mathrm{p}<0,001 *)$. Esta diferença foi mantida durante todo o seguimento, sendo estatisticamente significante nos momentos de uma semana ( $\mathrm{p}<0,001 *)$, um mês $\left(\mathrm{p}=0,008^{*}\right)$, seis $(\mathrm{p}=0,007 *)$ e 24 meses $(\mathrm{p}=0,002 *)$. Ao último exame, $\mathrm{AV} \mathrm{s} / \mathrm{c}$ de 20/20, ou melhor, foi observada em quatro dos 14 olhos $(28,57 \%)$ do grupo ICRS, e em 12 dos 14 olhos $(85,71 \%)$ do grupo LASIK $(\mathrm{p}=0,002 *)$. A porcentagem de olhos com AV s/c $\geq 20 / 40$ foi semelhante em ambos os grupos (92,86\% e 100\% para ICRS e LASIK, respectivamente $)(\mathrm{p}=1,000)$.

\section{Acuidade visual com correção}

Durante todo o seguimento, não foram observadas perdas $\geq$ duas linhas da melhor acuidade visual corrigida em ambos os grupos.

\section{Videoceratografia computadorizada}

O aplanamento corneano foi observado por meio da redução estatisticamente significante dos valores do poder dióptrico central da córnea em ambos os grupos. $\mathrm{O}$ aspecto prolado da córnea foi mantido em todos os olhos do grupo ICRS, ao contrário da superfície oblada observada no pós-operatório do grupo controle.

\section{Sensibilidade ao contraste}

Esta foi testada nos pós-operatórios de seis, 12 e 24 meses. Alterações médias da sensibilidade ao contraste em relação aos valores basais no grupo ICRS foram observadas ao exame de seis meses, nas freqüências espaciais de $12 \mathrm{cpd}\left(\mathrm{p}=0,011^{*}\right)$ e $18 \mathrm{cpd}\left(\mathrm{p}=0,007^{*}\right)$, tendo persistido durante as avaliações de 12 e 24 meses (Figura 4).

Redução estatisticamente significante da sensibilidade ao contraste no grupo controle, em relação aos valores pré-operatórios, só foi observada ao exame de seis meses, na frequiência espacial de $6 \mathrm{cpd}(\mathrm{p}=0,047 *)$ (Figura 5).

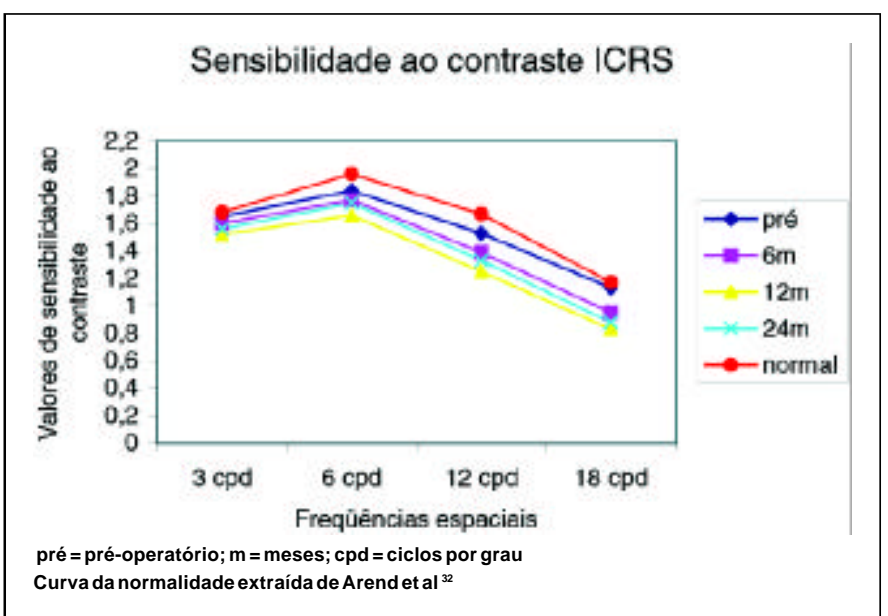

Figura 4 - Comportamento das curvas de sensibilidade ao contraste (em unidades de log) do grupo ICRS no pré-operatório e pós-operatórios de seis, 12 e 24 meses

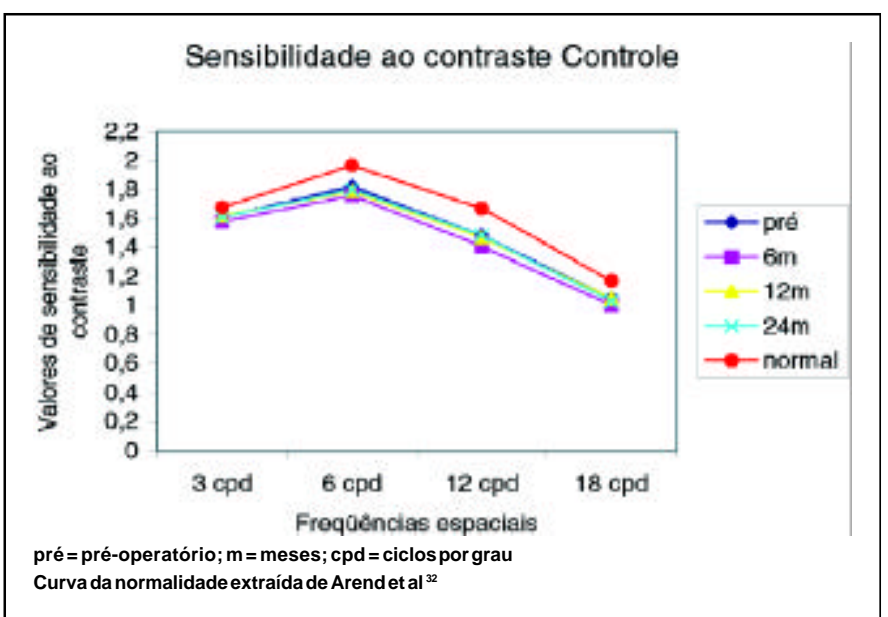

Figura 5 - Comportamento das curvas de sensibilidade ao contraste (em unidades de log) do grupo controle no pré-operatório e pósoperatórios de seis, 12 e 24 meses 
Diferenças estatisticamente significantes entre os dois grupos surgiram ao exame de 12 meses nas frequiências espaciais de $3 \mathrm{cpd}\left(\mathrm{p}=0,005^{*}\right) ; 6 \mathrm{cpd}\left(\mathrm{p}=0,018^{*}\right) ; 12 \mathrm{cpd}\left(\mathrm{p}=0,001^{*}\right)$ e $18 \mathrm{cpd}\left(\mathrm{p}=0,006^{*}\right) ; \mathrm{e}$, ao exame de 24 meses, nas frequiências de $12 \operatorname{cdp}\left(\mathrm{p}=0,045^{*}\right)$ e $18 \mathrm{cpd}(\mathrm{p}=0,032 *)$.

\section{Observações e complicações}

Opacidades nos canais estromais, ao redor dos segmentos de anéis, foram encontradas na maioria dos casos de ICRS no período pós-operatório precoce, persistindo em 13 olhos $(92,85 \%)$ ao exame de 24 meses.

Depósitos esbranquiçados dentro do canal estromal foram observados a partir do primeiro mês de pós-operatório, com aumento na freqüência e intensidade nos exames seguintes. No pós-operatório de 24 meses, os depósitos estavam presentes em 13 casos $(92,85 \%)$, sempre confinados ao canal estromal.

Linhas de depósito de ferro epiteliais foram observadas em 10 casos $(71,42 \%)$ ao exame de 24 meses, com aparecimento a partir do terceiro mês de cirurgia.

A migração dos segmentos de anéis para região inferior, observada pelo toque inferior entre os segmentos nasais e temporais, ocorreu em cinco casos $(35,71 \%)$.

Placas epiteliais nas incisões foram observadas em oito casos $(57,14 \%)$, entre os exames uma semana e três meses, com regressão espontânea na maioria destes.

Todos os seis olhos que não tiveram suas incisões suturadas ao término do procedimento cirúrgico, além de cinco inicialmente suturados, apresentaram indução de astigmatismo contra a regra ao exame de uma semana após a cirurgia, sendo esta superior a 1,00 D em nove olhos. Revisão cirúrgica da incisão, por meio do posicionamento de suturas com maior tensão, foi realizada em oito casos, entre os períodos pós-operatórios de uma semana e um mês, sendo que dois destes apresentavam placa epitelial na incisão associada (Figura 6).
Traços de ceratite lamelar difusa da interface foram encontrados em cinco olhos $(35,71 \%)$ do grupo controle ao exame de um dia, não tendo sido necessária mudança do esquema terapêutico previamente proposto.

\section{Questionário subjetivo}

Mais queixas subjetivas de visão de halos e baixa de acuidade visual noturna foram reportadas no pós-operatório precoce de ICRS em relação ao grupo controle (Tabela 2).

Todos os 14 pacientes responderam ao questionário de satisfação e preferência entregue nos retornos de 12 e 24 meses de cirurgia. Destes, apenas um $(7,14 \%)$ não notou diferença em relação ao intra-operatório entre as duas técnicas empregadas, sendo que os outros $13(92,85 \%)$ preferiram o LASIK. A técnica de LASIK também foi a preferida no período pósoperatório por 12 pacientes $(85,71 \%)$, sendo que dois $(14,28 \%)$ não relataram diferenças entre os procedimentos. A recuperação visual foi relatada como de ocorrência mais precoce no olho submetido ao LASIK por 11 pacientes $(78,57 \%)$, sendo que três $(21,42 \%)$ não referiram diferenças entre ambos os olhos. Em termos gerais, três pacientes $(21,42 \%)$ acharam que não havia diferença entre a sua visão final, comparando-se os dois olhos, e $11(78,57 \%)$ preferiram o olho do LASIK. As respostas fornecidas nos exames de 12 e 24 meses foram semelhantes.

\section{DISCUSSÃO}

A busca pelo procedimento cirúrgico mais eficaz e seguro possível é o objetivo de todo cirurgião refrativo, principalmente em se tratando de baixa miopia. Apesar das teóricas vantagens dos anéis corneanos apresentadas em diferentes publicações ${ }^{(8-9,12-14)}$, não existiam na literatura estudos controlados comparando esta

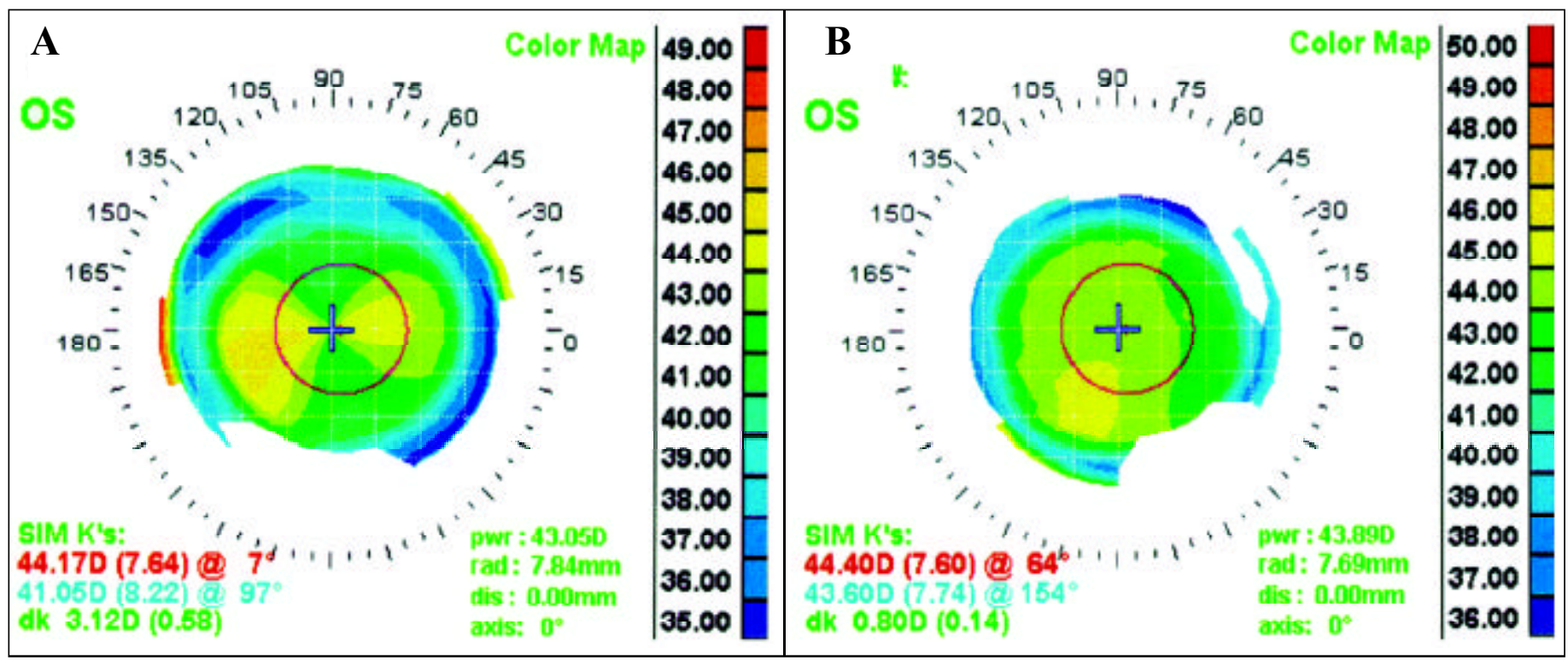

Figura 6 - Topografias corneanas de um paciente submetido à revisão cirúrgica da incisão na primeira semana de pós-operatório. A- pósoperatório de uma semana do implante de ICRS, antes da revisão da incisão; B- pós-operatório de três meses 


\begin{tabular}{|c|c|c|c|c|c|c|}
\hline \multirow[b]{2}{*}{ Sintomas } & \multicolumn{3}{|c|}{3 meses } & \multicolumn{3}{|c|}{24 meses } \\
\hline & ICRS & controle & p & ICRS & controle & $p$ \\
\hline Halos & $10(71,4 \%)$ & 0 & $<0,001^{*}$ & $8(57,1 \%)$ & $1(7,1 \%)$ & $0,013^{*}$ \\
\hline Diminuição da AV noturna & $9(64,3 \%)$ & $3(21,4 \%)$ & $0,022^{*}$ & $5(35,7 \%)$ & 0 & $0,041^{*}$ \\
\hline Flutuação da AV & $10(71,4 \%)$ & $1(7,1 \%)$ & $<0,001^{*}$ & 0 & 0 & - \\
\hline "Glare" & 0 & 0 & - & $4(28,6 \%)$ & 0 & 0,098 \\
\hline Embaçamento visual & $6(42,8 \%)$ & $2(14,3 \%)$ & 0,209 & 0 & 0 & - \\
\hline Olho seco & $4(28,6 \%)$ & $1(7,1 \%)$ & 0,326 & 0 & 0 & - \\
\hline Diplopia & $1(7,1 \%)$ & 0 & 1,000 & 0 & 0 & - \\
\hline
\end{tabular}

tecnologia com os procedimentos fotoablativos, mais comumente utilizados em pacientes com baixa miopia.

A possibilidade de incluir os olhos contralaterais dos pacientes operados por uma técnica cirúrgica já consagrada, o LASIK, permitiu o controle deste estudo. Críticas podem ser feitas quanto ao intervalo entre os dois procedimentos realizados, em média $6,27 \pm 1,94$ meses, mas este foi o período considerado necessário como critério de avaliação de estabilidade do olho submetido ao implante de ICRS. A bilateralidade fez com que os dois grupos fossem estatisticamente comparáveis em relação ao sexo e à idade. Semelhanças também foram observadas em relação a todas as características oftalmológicas avaliadas no período pré-operatório. A randomização não foi realizada pois o olho a ser implantado com ICRS deveria ser previamente selecionado, através do equivalente esférico da refração estática, de forma que a correção máxima pudesse ser obtida conforme nomograma refinado fornecido pelo fabricante. Outro aspecto importante foi a possibilidade de se ter um seguimento completo e padronizado de toda a pesquisa clínica desejada.

De acordo com este protocolo, o cirurgião poderia optar pelo não posicionamento de suturas na incisão, caso houvesse perfeita coaptação de suas bordas. Por este motivo, as seis primeiras cirurgias de ICRS não foram suturadas. Entretanto, devido ao astigmatismo contra a regra induzido, observado ao exame de uma semana de pós-operatório, optou-se pela sutura de todas as cirurgias subseqüentes.

\section{Refração}

No atual estudo, apesar de não haver uma distribuição homogênea dos pacientes em relação às diferentes espessuras dos anéis utilizados, a técnica de ICRS mostrou, de maneira geral, uma correção miópica média abaixo da prevista (Tabela 1). Hipocorreções foram observadas com os segmentos de 0,30 a $0,45 \mathrm{~mm}$ de espessura, no início da fase II de estudos do FDA, levando a um ajuste do nomograma ${ }^{(11)}$, para os padrões utilizados até hoje. A partir de então, maior concordância entre o efeito cirúrgico previsto para os anéis de $0,25,0,30$ e $0,35 \mathrm{~mm}$ de espessura e a correção média obtida foram reportadas ${ }^{(15-10)}$.

Apesar da hipocorreção observada, os critérios de eficácia em relação à previsibilidade do procedimento foram atingidos, uma vez que o efeito refrativo dentro da faixa de $\pm 1,00 \mathrm{D}$ da correção desejada em pelo menos $75 \%$ dos casos, ou entre $\pm 0,50 \mathrm{D}$ em $50 \%$, foi obtido. Este mesmo critério de previsibilidade foi observado em estudos de diferentes autores em relação à porcentagem de olhos entre $\pm 1,00 \mathrm{D}$ da correção desejada ${ }^{(11,15-19)}$, mas não entre $\pm 0,50 \mathrm{D}^{(11,18-19)}$ (Tabela 3 ).

A previsibilidade do efeito refrativo tende a diminuir com o aumento da espessura dos segmentos de anéis implantados ${ }^{(15-16)}$. A correlação negativa aqui encontrada mostrou que quanto maior a espessura do anel implantado, menor o valor do equivalente esférico da refração estática, ou seja, maior a miopia residual. Em outro estudo, $82 \%$ dos casos implantados com segmentos de $0,25 \mathrm{~mm}$ de espessura atingiram equivalente esférico entre $\pm 0,50 \mathrm{D}$ da correção desejada, contra $75 \%$ e $62 \%$ dos grupos de segmentos de $0,30 \mathrm{~mm}$ e $0,35 \mathrm{~mm}$ respectivamente $^{(16)}$. Pacientes implantados com segmentos de $0,35 \mathrm{~mm}$ tendem a apresentar piores resultados do que os implantados com segmentos de $0,25 \mathrm{~mm}$ e $0,30 \mathrm{~mm}$, e maior taxa de explante por insatisfação com os resultados obtidos ${ }^{(23)}$.

\begin{tabular}{|c|c|c|c|c|c|c|c|c|}
\hline Autores & $\begin{array}{l}\text { Espessura } \\
\text { ICRS (mm) }\end{array}$ & $\begin{array}{l}\text { Pós-op } \\
\text { (meses) }\end{array}$ & $\mathbf{n}^{*}$ & $\begin{array}{l}\text { AVs/c } \\
\geq 20 / 20\end{array}$ & $\begin{array}{l}\text { AVs/c } \\
\geq 20 / 40\end{array}$ & $\begin{array}{c}\text { EE } \\
\pm 0,50 \mathrm{D}\end{array}$ & $\begin{array}{c}\text { EE } \\
\pm 1,00 \mathrm{D}\end{array}$ & $\begin{array}{c}\text { Perda } \\
\geq 2 \mathrm{~L} \quad \mathrm{AVc} / \mathrm{c}\end{array}$ \\
\hline Schanzlin et al $(1997)^{11}$ & 0,25 a 0,45 & 3 & 102 & $54 \%$ & $96 \%$ & $41 \%$ & $77 \%$ & $5 \%$ \\
\hline Ruckhofer et al $(2000)^{18}$ & 0,30 a 0,45 & 24 & 30 & $73 \%$ & $90 \%$ & $47 \%$ & $90 \%$ & $0 \%$ \\
\hline Wijdh e Van Rij $(2000)^{17}$ & 0,25 a 0,45 & 6 & 21 & $43 \%$ & $100 \%$ & $81 \%$ & $100 \%$ & $0 \%$ \\
\hline Asbell e Uçakhan $(2001)^{15}$ & 0,21 a 0,45 & 3 a 36 & 113 & $72 \%$ & $95 \%$ & $63,7 \%$ & $92 \%$ & $0 \%$ \\
\hline Ruckhofer et al $(2001)^{19}$ & 0,25 a 0,45 & 12 & 159 & $63 \%$ & $96 \%$ & $49 \%$ & $82 \%$ & $6 \%$ \\
\hline Schanzlin et al $(2001)^{16}$ & 0,25 a 0,35 & 24 & 449 & $76 \%$ & $97 \%$ & $73 \%$ & $93 \%$ & $0,5 \%$ \\
\hline
\end{tabular}


Ao serem comparados os resultados obtidos com o grupo ICRS em relação ao controle, pôde-se observar que maior previsibilidade foi obtida por este último, no que se refere à porcentagem de olhos com equivalente esférico da refração estática dentro da faixa de $\pm 0,50 \mathrm{D}$ da correção desejada, apesar de esta diferença não ter sido considerada estatisticamente significante $(64,29 \%$ contra $85,71 \%)$. Menor previsibilidade do efeito refrativo no grupo ICRS também foi observada em estudo retrospectivo comparativo com a técnica de LASIK, acreditando estar relacionada à limitação da correção que pode ser obtida com os diferentes segmentos de anéis ${ }^{(21)}$. De acordo com o nomograma atual, cada aumento de $0,05 \mathrm{~mm}$ na espessura do implante a ser utilizado significa um efeito refrativo adicional em torno de $0,70 \mathrm{D}$, enquanto o "excimer laser" pode ser programado em incrementos de $0,01 \mathrm{D}$.

A estabilidade do efeito refrativo após cirurgia de ICRS pode ser atribuída ao próprio procedimento em si, que envolve o implante de um material biocompatível na córnea, cujo efeito não parece estar relacionado ao processo cicatricial de cada indivíduo $^{(12,15)}$. Asbell e Uçakhan consideraram o procedimento ICRS estável, com $96,7 \%$ dos casos apresentando alterações refracionais $\leq 0,50 \mathrm{D}$ entre os exames de três e seis meses, $97,4 \%$ entre seis e 12 meses, $94,8 \%$ entre 12 e 18 meses, e $97,1 \%$ entre 18 e 24 meses $^{(15)}$. Variações no equivalente esférico da refração dinâmica inferiores a $0,50 \mathrm{D}$ foram observadas por outros autores em $82 \%$ dos casos operados, entre os exames de três e seis meses, e por $80 \%$ dos olhos no intervalo de seis e 12 meses $^{(19)}$. O critério de estabilidade também foi obtido em estudo no qual, no mínimo, $97 \%$ e $82 \%$ dos casos operados apresentaram variação do equivalente esférico da refração dinâmica $\leq 1,00 \mathrm{D}$ e $\leq 0,50 \mathrm{D}$, respectivamente, a partir dos exames do terceiro mês pós-operatório ${ }^{(16)}$.

Neste estudo, os critérios de estabilidade foram obtidos por ambos os grupos. Críticas devem ser feitas quanto aos critérios aqui adotados: análise só a partir do terceiro mês, comparando-se dois exames consecutivos, e apenas o equivalente esférico. Isto porque várias alterações foram observadas antes do terceiro mês, principalmente no que se refere ao astigmatismo no grupo ICRS. O próprio grupo controle apresentou tendência à regressão a partir da primeira semana (Figura 1).

Os grupos ICRS e controle, que apresentavam comportamentos semelhantes no período pré-operatório em relação à média do astigmatismo da refração dinâmica, mostraram-se diferentes em todo o seguimento pós-operatório, às custas da maior indução de astigmatismo nos olhos implantados. De acordo com a literatura, indução de astigmatismo superior a 1,00 D pode ser observada entre $11 \%$ e $14,2 \%$ dos casos com ICRS $^{(15,19)}$. O astigmatismo, quando induzido, tende a regredir com o tempo ${ }^{(16)}$.

A causa dessa indução de astigmatismo ainda é desconhecida. Fatores como a migração dos segmentos de anéis dentro da canal estromal ${ }^{(24)} \mathrm{e}$ a tensão exagerada exercida pela sutura no local da incisão radial ${ }^{(15)}$ já foram propostos. É possível ainda que a incisão radial localizada na posição de 12 horas, associada à tensão exercida pelos segmentos nas regiões na- sais e temporais, exerça um efeito relaxante no meridiano em que ela foi posicionada, levando ao aplanamento deste local e indução de astigmatismo contra a regra como aqui observado. Segmentos de anéis mais espessos foram relacionados à maior indução de astigmatismo durante todo o acompanhamento pós-operatório, à semelhança de outro estudo ${ }^{(25)}$.

No grupo controle, o efeito cirúrgico máximo sobre a correção programada do astigmatismo foi observado no primeiro dia pós-operatório, com tendência à regressão após a primeira semana (Figura 2).

\section{Acuidade visual sem correção}

Embora o critério de eficácia na acuidade visual tenha sido atingido no grupo ICRS assim como em outros estudos (Tabela 3), melhores resultados foram obtidos no grupo controle, principalmente no que se refere a níveis de AV s/c de 20/20, ou melhor. Considerando-se este nível de acuidade visual como um dos fatores determinantes para menor necessidade do uso de correção óptica no pós-operatório ${ }^{(26)}$, os resultados obtidos com o implante de ICRS foram julgados insatisfatórios, em se tratando de pacientes portadores de baixa miopia. Esta diferença entre os dois grupos pode ser explicada pelo maior residual miópico observado no grupo ICRS.

Comparando-se os dois grupos, foi observado um atraso na recuperação visual com a técnica de ICRS, talvez ocasionado pela instabilidade refracional observada no pós-operatório precoce, relacionada principalmente à indução de astigmatismo. Em estudo comparativo retrospectivo entre as técnicas de LASIK e ICRS, também foi reportada maior demora na recuperação visual nesta última ${ }^{(21)}$. No primeiro dia pós-operatório, maior porcentagem de olhos após LASIK do que após ICRS tinha acuidade visual sem correção $\geq 20 / 40$ (95\% contra $87 \%), \geq 20 /$ 20 (55\% contra $24 \%)$, e $\geq 20 / 16$ ( $14 \%$ contra $6 \%$ ). Entretanto houve reversão desta tendência com o tempo, sendo que no terceiro mês pós-operatório mais casos submetidos à ICRS do que ao LASIK apresentavam AV s/c $\geq 20 / 40$ (99\% contra 95\%), $\geq 20 / 20$ (75\% contra $67 \%$ ) e $\geq 20 / 16$ (38\% contra $29 \%)$.

\section{Acuidade visual com correção}

Durante todo o estudo desta série nenhum paciente apresentou perda de duas ou mais linhas da melhor acuidade visual corrigida, demonstrando a segurança dos procedimentos ICRS e LASIK. A manutenção da acuidade visual corrigida no grupo ICRS pode ser atribuída à preservação da região central da córnea. Mesmo assim, outros autores reportaram uma taxa inesperada de perda $\geq$ duas linhas em $6 \%$ dos casos implantados com ICRS, ao exame de 12 meses, sendo que segmentos mais espessos haviam sido implantados na maioria destes olhos ${ }^{(19)}$.

\section{Sensibilidade ao contraste}

A medida da sensibilidade ao contraste reflete a função visual de maneira mais precisa do que a avaliação isolada da acuidade visual em alto contraste ${ }^{(27)}$. A manutenção do aspecto asférico prolado da córnea parece ser importante na preservação visual funcional no período pós-operatório de um procedimento refrativo ${ }^{(12)}$. 
Após a cirurgia de ICRS, a córnea tende a tornar-se mais prolada em relação ao seu aspecto pré-operatório ${ }^{(28)}$, principalmente com implantes de segmentos mais espessos, já tendo sido demonstrada piora da função visual em alguns olhos com asfericidade pós-operatória muito elevada ${ }^{(29)}$. Apesar de não se ter analisado quantitativamente as alterações da asfericidade corneana no atual estudo, o grupo ICRS apresentou redução da média da sensibilidade ao contraste nas frequiências espaciais mais altas ( 12 cpd e 18 cpd) ao exame de seis meses, alteração esta que persistiu nas avaliações de 12 e 24 meses. Portanto é possível que o aumento desta asfericidade também possa influenciar negativamente o desempenho visual dos pacientes. Redução da sensibilidade ao contraste até o terceiro mês após o implante de ICRS já foi relatada ${ }^{(24)}$, contrariamente a outros estudos nos quais não foram encontradas alterações estatisticamente significantes aos exames de três, seis e doze meses de cirurgia ${ }^{(11,15)}$.

Por outro lado, no grupo controle, as alterações da sensibilidade ao contraste não foram tão evidentes, demonstrando melhor recuperação do desempenho visual. Maiores reduções poderiam ter sido diagnosticadas se estes pacientes tivessem sido analisados em períodos pós-operatórios mais precoces, como um ou três meses.

No pós-operatório de seis meses os dois grupos mostraram-se semelhantes em relação às curvas de sensibilidade ao contraste, sendo que a partir de então surgiram diferenças estatisticamente significantes, principalmente às custas da melhora da função visual observada no grupo controle, não acompanhada pelo grupo ICRS. Até o presente momento não foram encontrados estudos na literatura comparando as técnicas de ICRS e LASIK em relação à sensibilidade ao contraste no pós-operatório. Em estudo comparativo entre ICRS e PRK foi observada redução da sensibilidade ao contraste nas freqüências espaciais de 6 e $12 \mathrm{cpd}$, principalmente com "glare", em ambos os procedimentos ao exame de três meses, sem diferenças significantes entre eles, com tendência à normalização após este período ${ }^{(20)}$.

\section{Observações e complicações}

As opacidades no canal estromal, os depósitos esbranquiçados e as linhas de depósito de ferro na córnea do grupo ICRS já foram reportadas por outros autores ${ }^{(8-11,15-16,30)}$, sendo classificadas como observações oculares e consideradas sem significado clínico, necessitando apenas de acompanhamento.

A migração dos segmentos de anéis dentro do canal estromal foi encontrada em cinco casos $(35,71 \%)$ implantados, à semelhança de outro estudo ${ }^{(24)}$. Cuidados devem ser tomados no sentido de evitar a união dos dois semicanais durante a dissecção cirúrgica lamelar, o que reduziria o contato inferior dos dois segmentos. Isto porque maior astigmatismo pós-operatório foi observado em pacientes que apresentaram migração inferior dos segmentos ${ }^{(24)}$. É possível que a manutenção de uma pequena distância entre as extremidades inferiores dos ICRS faça com que a tensão seja distribuída de maneira mais uniforme por toda a circunferência da córnea, reduzindo o risco de indução de astigmatismo.

A revisão cirúrgica da incisão, por meio do posicionamento de suturas com maior tensão, foi necessária em oito casos em função da presença de astigmatismo contra a regra, associado ou não à placa epitelial na incisão. Placas epiteliais já foram relatadas em $11 \%$ dos casos implantados com ICRS, com resolução até o sexto mês, à exceção de um ${ }^{(30)}$. Parece que o posicionamento das suturas permite o maior controle da aproximação das bordas da incisão, reduzindo o risco de ocorrência de placas ou cistos epiteliais ${ }^{(20)}$.

Casos com indução de astigmatismo a favor da regra no pós-operatório de ICRS podem ser beneficiados pelo reaprofundamento da incisão ${ }^{(20)}$. Deiscência da incisão foi descrita em um paciente implantado com segmentos de $0,45 \mathrm{~mm}$, após remoção das suturas na segunda semana de cirurgia, sendo necessário seu reposicionamento ${ }^{(15)}$.

A presença de ceratite lamelar difusa da interface em cinco olhos operados pela técnica de LASIK, apesar de discreta, foi considerada como complicação ocular por ter representado um achado com risco potencial de se tornar clinicamente significante. Quadros extremos de ceratite lamelar difusa da interface já foram relacionados à perda da melhor acuidade visual corrigida ${ }^{(31)}$, sendo grande a preocupação quanto à identificação dos possíveis fatores etiológicos envolvidos, na tentativa de prevenir o aparecimento da referida situação.

\section{Questionário subjetivo}

Neste estudo, a flutuação da acuidade visual, visão de halos e dificuldades para enxergar à noite foram as principais queixas referidas no pós-operatório de três meses no grupo ICRS, com tendência à regressão nas demais avaliações, tanto em intensidade quanto em freqüência. Sintomas subjetivos como os aqui reportados também foram relatados durante a fase II de estudos do FDA, tendo sido responsável pela maioria das indicações de explante (20 dos 37 casos explantados) ${ }^{(16)}$.

De maneira geral, o grupo controle apresentou menores queixas subjetivas em relação ao ICRS em todas as avaliações pós-operatórias. Queixas de halos e "glare" podem ocorrer no pós-operatório de LASIK em condições em que a pupila apresente-se com diâmetro maior do que a zona de tratamento. Um estudo comparativo entre as técnicas de PRK e ICRS reportou freqüência semelhante de sintomas visuais nos dois grupos de pacientes ${ }^{(20)}$. O perfil de evolução do decréscimo desses sintomas foi mais rápido nos olhos operados pela técnica de PRK.

A técnica de LASIK foi a preferida pela maioria dos pacientes, podendo ser o reflexo do menor tempo cirúrgico necessário para a realização do procedimento, da recuperação visual mais precoce, e do menor residual miópico observado.

As diferenças observadas pelos pacientes quanto à acuidade visual final refletem o que foi encontrado em relação à porcentagem de olhos com visão $\geq 20 / 20$, ao último exame pósoperatório. Nesta ocasião, este nível de acuidade visual foi obtido em quatro olhos operados pela técnica ICRS e 12 pela de LASIK, em concordância com a maior preferência dos pacientes por este último procedimento.

Não obstante as diferenças observadas entre os dois grupos, em nenhum momento foi solicitado o explante dos segmentos de anéis corneanos intra-estromais por parte dos pacientes, 
possibilidade esta previamente discutida com cada um deles no início do protocolo deste estudo.

Em resumo, os segmentos de anéis corneanos intraestromais foram considerados eficazes na correção de baixa miopia, porém sua segurança foi limitada pela indução de astigmatismo observada no pós-operatório. Os resultados obtidos foram inferiores aos do grupo controle, embora essa diferença não tenha sido considerada estatisticamente significante de acordo com este protocolo. Devido às limitações de indicação da técnica de implante de segmentos de anéis corneanos intra-estromais associada à necessidade de maior habilidade cirúrgica, ao contrário do que ocorre com os procedimentos fotoablativos como PRK e LASIK, fica difícil imaginar uma reversão da preferência por parte dos cirurgiões no que se refere ao tratamento cirúrgico da baixa miopia.

\section{ABSTRACT}

Purpose: To evaluate the efficacy and safety of intrastromal corneal ring segments (ICRS) for the correction of low myopia, and to compare the obtained results with a control group submitted to laser in situ keratomileusis (LASIK). Methods: Prospective, nonrandomized and bilateral study. Fourteen patients with preoperative spherical equivalent ranging from 1.00 to -4.50 D received ICRS in one eye and LASIK in the other. Postoperative evaluations were performed at day one and seven and months 1, 3, 6, 12 and 24. Results: At the last follow-up, $64.29 \%$ of ICRS eyes (9/14) and $85.71 \%$ of LASIK eyes (12/14) were within $\pm 0.50 \mathrm{D}$ of the predicted refractive outcome $(\mathrm{p}=0.385)$. Uncorrected visual acuity of $20 / 20$ or better was observed in four of 14 ICRS eyes $(28.57 \%)$, and in 12 of 14 LASIK eyes $(85.71 \%)(\mathrm{p}=0.002 *)$. No variances of more than $1.00 \mathrm{D}$ in the manifest spherical equivalent refraction between two consecutive examinations or significant loss of best spectacle-corrected visual acuity were observed in both groups. Surgically induced astigmatism was greater in ICRS eyes than in LASIK eyes. At 24 months, the ICRS eyes scored lower in contrast sensitivity testing, especially at higher spatial frequencies $(\mathrm{p}=0.032 *$ at $18 \mathrm{cpd})$. The patients reported more subjective complaints in the early postoperative period for the ICRS eyes. Conclusions: ICRS were considered effective for the correction of low myopia, but their safety was limited by the observed surgically induced astigmatism, and their results were inferior compared to the control group.

Keywords: Myopia/surgery; Cornea/surgery; Prosthesis implantation; Laser in situ keratomileusis; Myopia/classification

\section{REFERÊNCIAS}

1. Barraquer JI. Queratoplastia refractiva. Est e Inf Oftal 1949;2:10-30.

2. Barraquer JI. Modification of refraction by means of intracorneal inclusions. Int Ophthalmol Clin 1966;6:53-78.
3. McCarey BE, Andrews DM. Refractive keratoplasty with intrastromal hydrogel lenticular implants. Invest Ophthalmol Vis Sci 1981;21:107-15.

4. Choyce DP. The correction of refractive errors with polysulfone corneal inlays. A new frontier to be explored? Trans Ophthalmol Soc U K 1985;104:332-42.

5. Simon DG, Barraquer RI. Queratocricoemfitesis: nuevo procedimiento de cirugia refractiva (estudio experimental). Arch Soc Esp Oftalmol Invest 1988;1:87-94.

6. Fleming JF, Reynolds AE, Kilmer L, Burris TE, Abott RL, Schanzlin DJ. The intrastromal corneal ring: Two cases in rabbits. J Refract Surg 1987;3:227-32.

7. Fleming JF, Wan WL, Schanzlin DJ. The theory of corneal curvature change with the intrastromal corneal ring. CLAO J 1989;15:146-50.

8. Nosé W, Neves RA, Schanzlin DJ, Belfort Júnior R. Intrastromal corneal ring one-year results of first implants in humans: a preliminary nonfunctional eye study. Refract Corneal Surg 1993;9:452-8.

9. Nosé W, Neves RA, Burris TE, Schanzlin DJ, Belfort Júnior R. Intrastromal corneal ring: 12-month sighted myopic eyes. J Refract Surg 1996;12:20-8.

10. Assil KK, Barrett AM, Fouraker BD, Schanzlin DJ. One-year results of the intrastromal corneal ring in nonfunctional human eyes. Intrastromal corneal ring Study Group. Arch Ophthalmol 1995;113:159-67.

11. Schanzlin DJ, Asbell PA, Burris TE, Durrie DS. The intrastromal corneal ring segments. Phase II results for the correction of myopia. Ophthalmology 1997; 104:1067-78.

12. Krueger RR, Burris TE. Intrastromal corneal ring technology. Int Ophthalmol Clin 1996;36:89-106

13. Asbell PA, Uçakhan OO, Abbott RL, et al. Intrastomal corneal ring segments: reversibility of refractive effect. J Refract Surg 2001;17:25-31.

14. Asbell PA, Uçakhan OO, Durrie DS, Lindstrom RL. Adjustability of refractive effect for corneal ring segments. J Refract Surg 1999;15:627-31.

15. Asbell PA, Uçakhan OO. Long-term follow-up of Intacs from a single center. J Cataract Refract Surg 2001;27:1456-68.

16. Schanzlin DJ, Abbott RL, Asbell PA, Assil KK, Burris TE, Durrie DS, et al. Two-year outcomes of intrastromal corneal ring segments for the correction of myopia. Ophthalmology 2001;108:1688-94.

17. Wijdh RH, van Rij G. Intrastromal corneal ring segments (ICRs): three - and six months results. Doc Ophthalmol 2000;100:27-37.

18. Ruckhofer J, Stoiber J, Alzner E, Grabner G. Intrastromal corneal ring segments (ICRS, KeraVision Ring, Intacs): clinical outcome after 2 years. Klin Monatsbl Augenheilkd 2000;216:133-42.

19. Ruckhofer J, Stoiber J, Alzner E, Grabner G. One-year results of European Multicenter Study of intrastromal corneal ring segments. Part 1: refractive outcomes. J Cataract Refract Surg 2001;27:277-86.

20. Cochener B, Le Floch-Savary G, Colin J. Excimer photorefractive keratectomy (PRK) versus intrastromal corneal ring segments (ICRS) for correction of low myopia. J Fr Ophtalmol 2000;23:663-78.

21. Suiter BG, Twa MD, Ruckhofer J, Schanzlin DJ. A comparison of visual acuity, predictability, and visual function outcomes after intracorneal ring segments and laser in situ keratomileusis. Trans Am Ophthalmol Soc 2000;98:51-5.

22. Alpins NA. A new method of analyzing vectors for changes in astigmatism. J Cataract Refract Surg 1993;19:524-33.

23. Rapuano CJ, Sugar A, Koch DD, Agapitos PJ, Culbertson WW, de Luise VP, et al. Intrastromal corneal ring segments for low myopia: a report by the American Academy of Ophthalmology. Ophthalmology 2001;108:1922-8.

24. Cochener B, Savary-LeFloch G, Colin J. Effect of intrastromal corneal ring segment shift on clinical outcome: one year results for low myopia. J Cataract Refract Surg 2000;26:978-86.

25. Twa MD, Ruckhofer J, Shanzlin DJ. Surgically induced astigmatism after implantation of intacs intrastromal corneal ring segments. J Cataract Refract Surg 2001; 27:411-5

26. Bourque LB, Lynn MJ, Waring GO, Cartwright C. Spectacle and contact lens wearing six years after radial keratotomy in the Prospective Evaluation of Radial Keratotomy Study. Ophthalmology 1994;101:421-31.

27. Montés-Micó R, Charman WN. Choice of spatial frequency for contrast sensitivity evaluation after corneal refractive surgery. J Refract Surg 2001;17:646-51.

28. Holmes-Higgin DK, Baker PC, Burris TE, Silvestrini TA. Characterization of the aspheric corneal surface with intrastromal corneal ring segments. J Refract Surg 1999;15:520-8.

29. Holmes-Higgin DK, Burris TE. Corneal surface topography and associated visual performance with INTACS for myopia: phase III clinical trial results. The INTACS Study Group. Ophthalmology 2000;107:2061-71.

30. Ruckhofer J, Stoiber J, Alzner E, Grabner G. One-year results of European Multicenter Study of intrastromal corneal ring segments. Part 2: complications, visual symptoms, and patient satisfaction. J Cataract Refract Surg 2001; 27:287-96.

31. Parolini B, Marcon G, Panozzo GA. Central necrotic lamellar inflammation after laser in situ keratomileusis. J Refract Surg 2001;17:110-2.

32. Arend O, Remky A, Evans D, Stüber R, Harris A. Contrast sensitivity loss is coupled with capillary dropout in patients with diabetes. Invest Ophthalmol Vis Sci 1997;38:1819-24.

\footnotetext{
* Significant
} 